
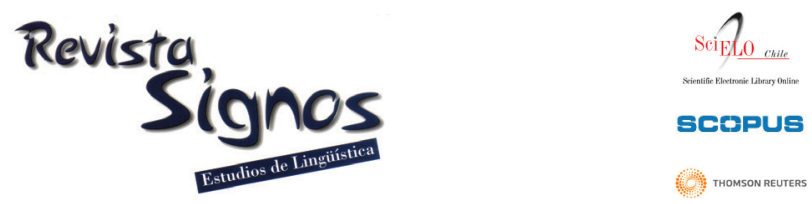

\title{
Los marcadores discursivos como rasgo distintivo de los textos del ámbito de las ciencias jurídicas en lengua vasca: Aproximación estadística con proyección jurilingüística
}

\section{Discourse markers as a distinctive feature of Basque legal texts: $A$ statistical approach applicable to legal language studies}

\author{
Joseba Ezeiza Ramos \\ Universidad del País Vasco \\ (UPV/EHU) \\ joseba.ezeiza@ehu.es
}

\section{Xabier Alberdi Larizgoitia}

Universidad del País Vasco (UPV/EHU)

xabier.alberdi@ehu.es

Recibido: 17-III-2013 / Aceptado: 23-III-2014

\section{Resumen}

En este trabajo se ha investigado qué factores podrían influir en la distribución y frecuencia de uso de los marcadores discursivos en los textos en euskera del ámbito de las ciencias jurídicas. Para ello, se ha realizado un estudio cuantitativo basado en la metodología de corpus, y se han aplicado técnicas de estadística inferencial para establecer el grado de significatividad de los patrones y diferencias observados. Los resultados obtenidos revelan varios hallazgos de interés teórico y aplicado. En primer lugar, los datos de frecuencia de uso parecen correlacionar de forma consistente con el grado de especialización de los textos y con el ámbito temático al que dichos textos pertenecen. Del mismo modo, se ha podido comprobar que los textos normativos y los textos académicos presentan configuraciones significativamente diferenciadas, lo cual puede tener relación con el registro propio de unos y otros. También se ha podido concluir que ciertos rasgos distintivos propios de algunas de las variedades de textos académicos analizadas pueden estar relacionados con las especificidades de los géneros textuales considerados. Finalmente, se ha podido comprobar que aun cuando la autoría de las traducciones de los textos normativos parece determinar ciertos patrones distintivos de estilo, por lo que al uso de marcadores discursivos se refiere, los datos sugieren una tendencia convergente. Esta conclusión resulta especialmente alentadora, dada la situación sociolingüística del euskera en el ámbito jurídico, en el que su uso no se encontraría aún completamente normalizado.

Palabras Clave: Jurilingüística, lenguaje jurídico, marcadores del discurso, lingüística de corpus, lengua vasca. 


\begin{abstract}
This paper presents a research project which analyses the frequency, distribution and patterning of discourse markers. The results point to substantially different tendencies: a) in texts in the domain of legal studies, in comparison to those found in journalistic, literary, humanities or science texts; b) in legal texts in comparison to academic law texts; c) in texts produced by different bodies currently involved in the development of Basque legal prose in comparison to each other. In its methodology the study is quantitative, and its findings appear to confirm its initial hypotheses. One of these is that frequencies of use seem to correlate clearly and consistently with the grade of specialization and the thematic domain of the texts. Another hypothesis states that the differences are highly significant in the case of the texts in the domain of legal studies, which seems to indicate that the frequency of use of makers shows relevant differences at register and genre level configurations between academic and legislative texts. Lastly, in the matter of the choice and frequency of discourse markers there seems to be a trend towards a common legislative style, shared by the various agencies and institutions involved in translating legal texts to Basque. This is encouraging, given the sociolinguistic situation of the Basque language in this area.
\end{abstract}

Key Words: Legal language studies; jurisprudence; discourse markers; corpus linguistics; Basque language.

\title{
INTRODUCCIÓN
}

En el contexto de los estudios vascos sobre marcadores del discurso, nuestra aportación se sitúa en el eje de los estudios de variación. Tal y como indican Loureda y Acín (2010), parece una hipótesis razonable que en variedades textuales diferentes se espere observar, desde el punto de vista cualitativo, el predominio de una u otra función de los marcadores discursivos y que, desde un punto de vista cuantitativo, puedan existir diferencias en la frecuencia de uso de los distintos marcadores. Partiendo de esta idea, nuestro estudio pretende investigar qué factores podrían influir en los patrones de selección y uso de marcadores discursivos observados en los textos en euskera del ámbito de las ciencias jurídicas.

En primer lugar, se ha analizado si existe algún tipo de correlación entre el carácter especializado de los textos de dicho ámbito y las frecuencias de uso de los principales marcadores discursivos, y, también, si las especificidades del dominio temático podrían condicionar dichas frecuencias de uso. En segundo lugar, el trabajo ha centrado específicamente la atención en los textos del ámbito disciplinar de las ciencias jurídicas, y se ha estudiado si se aprecian diferencias significativas en las frecuencias de uso de marcadores discursivos en dos variedades textuales que responden a fines comunicativos diferenciados, y que, por ello, se presume que puedan presentar diferencias a nivel de registro; se trataría de los textos normativos (códigos, 
leyes generales y leyes autonómicas) y los textos académicos de nivel universitario (monografías, manuales universitarios y artículos doctrinales). Finalmente, se ha focalizado la atención en los textos normativos, y se ha analizado el grado de sistematicidad y convergencia de la distribución de los marcadores discursivos en los textos traducidos al euskera por diferentes organismos e instituciones. Ello ha permitido estudiar la posible influencia de la autoría de las traducciones en los criterios de selección y uso de marcadores.

Desde el punto de vista metodológico, se trata de un estudio cuantitativo basado en el análisis de corpus amplios y diversos. Para tratar los datos se ha aplicado la estadística inferencial. Evidentemente, las conclusiones de este tipo de trabajos son limitadas y deben ser complementadas con investigaciones cualitativas y onomasiológicas como, por ejemplo, las de Landone (2010), Mazzi (2011), Alberdi y Ezeiza (2013) o Ezeiza (2014).

\section{Marco teórico}

\subsection{Contexto del estudio: Desarrollo funcional del euskera estándar; norma y uso}

El euskera es una lengua no indoeuropea hablada en el País Vasco que cuenta hoy con más de 700.000 hablantes repartidos a ambos lados de la frontera francoespañola, la cual constituye el límite político-administrativo entre el País Vasco peninsular (situado en territorio español) y el País Vasco continental (situado en territorio francés).

Por lo que respecta a su estatus, el euskera es lengua cooficial en la Comunidad Autónoma del País Vasco y en la zona declarada vascófona de la Comunidad Foral Navarra; pero no goza de tal estatus en la zona vasco-francesa. Por otra parte, se trata de una lengua que presenta una gran dispersión dialectal y que históricamente no ha contado con un modelo estándar común hasta una etapa reciente. Concretamente, es en 1968 cuando, en el congreso de la Real Academia de la Lengua Vasca (Euskaltzaindia), se sientan las bases de la unificación (Salaburu \& Alberdi, 2012). Gracias a ello, entre 1968 y 1990 el euskera se extiende a ámbitos como la enseñanza, la administración y los medios de comunicación, y ello trae consigo un rápido desarrollo funcional de la lengua, que, a su vez, genera la necesidad de nuevos registros y de nuevos recursos léxico-discursivos.

Es en ese contexto en el que hay que enmarcar el desarrollo del euskera jurídico, un registro que apenas tiene precedentes históricos y que, tras un breve período durante el primer Gobierno Vasco (1936-1937), se desarrolla principalmente de forma paralela al proceso de estandarización de la lengua común tras la restitución en 1979 del Gobierno Vasco y la publicación, a partir de 1980, del Boletín Oficial del País Vasco. Hoy en día el euskera jurídico tiene una presencia relevante en el País Vasco 
peninsular en los estudios universitarios de derecho y en la administración, y un espacio más limitado en la administración de justicia y en el ámbito legislativo. No obstante, a día de hoy el euskera se está consolidando como lengua de uso en todos estos ámbitos ${ }^{1}$.

Uno de los problemas notorios a los que se enfrenta el desarrollo del euskera jurídico se refiere a la terminología, dado que el desarrollo del léxico especializado se produce simultáneamente a la estandarización del léxico general. Pero junto a esas nuevas necesidades léxicas, surgen también nuevas necesidades discursivas: se trata de alcanzar, más allá de la norma, la idoneidad o adecuación necesarias en la comunicación especializada, y en ese contexto se toma conciencia de la importancia que tienen los marcadores discursivos para construir textos más adecuados y más 'comunicativos'. Actualmente la norma es considerablemente amplia en lo que respecta a los marcadores del discurso. De hecho, se cuenta con una descripción panhistórica de algunos de los principales marcadores discursivos, elaborada por la Academia de la Lengua Vasca (Euskaltzaindia, 1990). También se dispone de un Diccionario Unificado (Euskaltzaindia, 2000) que recoge ya la forma estándar de un buen número de marcadores. También se cuenta con el Diccionario de Euskaltzaindia (Euskaltzaindia, 2012). No obstante, aún no se puede considerar que el uso de los marcadores discursivos se haya fijado ni en la lengua estándar, ni tampoco en los registros especializados. Esto afecta también a los textos jurídicos, por lo cual, ofrecen un interesante campo de investigación.

\subsection{Los marcadores del discurso en euskera: Aproximación estratégica}

Hasta una etapa relativamente reciente los marcadores del discurso parecían caer en una especie de tierra de nadie en los estudios lingüísticos. Debido a su carácter transoracional y discursivo no constituían objeto de estudio para los gramáticos; ni tampoco lo eran para los lexicógrafos, que, en el mejor de los casos, resolvían la cuestión de su uso mediante sinónimos (Vázquez Veiga, 2011). Los estudios vascos sobre estos elementos no podían sustraerse a ese contexto general. Es precisamente en 1990 cuando la Real Academia de la Lengua Vasca (Euskaltzaindia, 1990) publica un tomo de su gramática descriptiva dedicado a los conectores - eusk. lokailuak-. Como en otras lenguas, el inicio del estudio de los marcadores discursivos surge a partir del análisis de la sintaxis oracional, cuando se toma conciencia de que estas unidades transcienden el nivel de la oración (Loureda \& Acín, 2010). Sin embargo, hay que subrayar que en este trabajo de Euskaltzaindia la perspectiva dominante es la gramatical. De hecho, habrá que esperar unos años para encontrar la primera aproximación discursiva a los marcadores: en 1996 se presenta en la Universidad del País Vasco (UPV/EHU) -sin llegar a publicarse- una tesis doctoral (Larringan, 1996) que analiza el uso de los 'organizadores textuales' (testu-antolatraileak) en textos informativos y argumentativos. 
Actualmente siguen siendo contados los trabajos descriptivos o teóricos sobre los marcadores desde una perspectiva discursiva (Esnal, 2002; Aierbe, 2009; Alberdi, 2011a, 2011b; Casado \& Barandiaran, 2011; Alberdi \& Ezeiza, 2013; Alberdi, 2014; Ezeiza, 2014; etc.), pero durante esta última década se han producido algunas aproximaciones relevantes a este objeto de estudio. Entre ellas cabe citar un trabajo de la Academia de la Lengua Vasca (Euskaltzaindia, 2008) que pone el foco de atención en los marcadores del discurso, situando como horizonte la mejora de la calidad lingüística de los textos ('idoneidad comunicativa'). De hecho, actualmente existe unanimidad en los diversos ámbitos profesionales y académicos vascos -y más particularmente en los de la administración y la justicia (Lozano, 1997; Urrutia, 2008) - en torno a la posible contribución de los marcadores discursivos a calidad de los textos y de la comunicación.

Sin embargo, no se aprecia el mismo grado de consenso por lo que respecta a la conceptualización, y a las terminologías empleadas: eusk. lokailu 'conector'; testu-antolatzaile 'organizador textual'; testu-markatzaile 'marcador textual'; diskurtso-markatzaile 'marcador discursivo'; etc. Fuera del ámbito del euskera, también es sorprendente la cantidad de denominaciones ${ }^{2}$ utilizadas para designar a estos recursos lingüísticos (Martín Zorraquino \& Portolés, 1999; Garcés, 2008; Loureda \& Acín, 2010; Vázquez Veiga, 2011). En cualquier caso, en este trabajo hacemos nuestra la definición propuesta por Martín Zorraquino y Portolés (1999), la cual subraya la función procedimental ${ }^{3}$, instruccional o de procesamiento (Blakemore, 2002; Portolés, 2007) de los marcadores, entendida como una orientación cooperativa en la interpretación del enunciado (Aschenberg \& Loureda, 2011). De acuerdo con esta perspectiva, los marcadores constituirían una categoría funcional, discursiva o pragmática (Loureda \& Acín, 2010; Real Academia Española, Asociación de Academias de la Lengua Española, 2010; Martí Sánchez, 2011). Así pues, nos parece fundamental insistir en el hecho de que una característica fundamental de los marcadores es su función procedimental de facilitar y guiar en la interpretación de los enunciados. Es decir, sin poner en duda la aportación del enfoque que predomina en algunos estudios vascos centrados en ciertas opciones estratégicas concretas del empleo de los marcadores del discurso (Euskaltzaindia, 2008), queremos subrayar el valor 'estratégico' inmanente de su uso.

\subsection{Los marcadores del discurso en perspectiva jurilingüística}

La perspectiva jurilingüística (Gemar, 1982; Gemar \& Kasirer, 2005; Urrutia, 2005; Ezeiza, 2011a) propone una aproximación dual al discurso jurídico en la que se conjugue una mirada al derecho desde la lingüística con una mirada al lenguaje desde el derecho. Con ello se trataría de plantear soluciones que optimicen dos criterios que no siempre son sencillos de conciliar: el de la eficacia comunicativa y el de la seguridad jurídica. Precisamente, con respecto a los textos jurídicos elaborados en euskera existe una opinión compartida por juristas, traductores y lingüistas que coincide en atribuir 
a los marcadores discursivos un valor estratégico para el desarrollo de un lenguaje jurídico que responda al reto que plantea el conocido como 'derecho a comprender's4.

De hecho, la tesis de que los marcadores discursivos tienen una función procedimental, instruccional o de procesamiento parece ofrecer un soporte suficiente para postular una presumible contribución de su uso estratégico al incremento de la eficacia comunicativa y, consecuentemente, al refuerzo de la seguridad jurídica. En el caso del euskera, el interés por el estudio de los marcadores tendría una justificación adicional. De hecho, la mayor parte de los textos jurídicos en euskera son traducidos, y existe la posibilidad de que la selección y uso de los marcadores pueda responder, en ocasiones, a meras estrategias oportunistas concebidas para resolver problemas puntuales de traducción.

Dado que es posible que los diversos agentes implicados en la elaboración de traducciones y de documentos bilingües no apliquen dichas estrategias de forma sistemática y regular, puede paradójicamente suceder que, el uso de marcadores contribuya de forma puntual a incrementar la eficacia comunicativa de los textos, pero que, al mismo tiempo, su seguridad jurídica quede comprometida por la aplicación asistemática de dichas estrategias. De ello se deduce que la investigación del uso de marcadores en los textos de este ámbito trascendería el interés meramente lingüístico, y que podría proyectar implicaciones de tipo jurídico.

\subsection{Modelo teórico: Aproximación desde la visión sociocognitiva del discurso, el variacionismo y la lingüística de corpus}

Partiendo de las premisas anteriormente señaladas, y asumiendo la proyección jurilingüística del problema de investigación, se ha optado por abordar el estudio desde una visión sociocognitiva del discurso (Bhatia, 1993, 2002; Bhatia \& Bhatia, 2011; etc.). De hecho, de acuerdo con Bhatia (2002), la visión sociocognitiva del discurso focalizaría la atención en la interfaz a la que concurrirían, por un lado, la dimensión del discurso como texto y, por otro, su proyección como práctica social. De esta concurrencia emergería un conocimiento (denominado por Bhatia genre knowledge) acerca de la forma en que se construyen los textos, y también acerca de la forma en que se interpretan, se reproducen o adaptan, y los efectos que estos producen en determinados contextos sociales (Bhatia, 2002). Desde este punto de vista, un objetivo como el trazado en nuestra investigación debería abordarse a través del análisis de los textos que generan las prácticas de la comunidad discursiva por la que estamos interesados, prestando atención a los factores sociocognitivos que pudieran influir en los criterios de selección y uso de los recursos lingüísticos que constituyen dichos textos.

Por otra parte, comoquiera que, siguiendo a Loureda y Acín (2010), esta investigación parte de la hipótesis de que es posible que diferentes variedades 
de textos puedan presentar diferencias cuantitativas y cualitativas en el uso de marcadores, la perspectiva que parece más coherente con el fin pretendido, la ofrecería una aproximación al discurso desde la variación (Biber, 1988, 1995, 2006; Biber \& Finegan, 1994; Conrad \& Biber, 2001; Biber \& Conrad, 2011; etc.). De hecho, de acuerdo con este punto de vista, los patrones lingüísticos representativos de los textos 'no serían arbitrarios' (Biber \& Conrad, 2011), y responderían a "estrechos vínculos con el contexto extralingüístico" (Petöfi, 1981, citado en Ciapuscio, 2003: 29). Así pues, los textos se caracterizarían por "una configuración global específica" (Cabré \& Gómez de Enterría, 2006: 23), pero que "manifestaría variaciones según la temática y los distintos niveles en la que se trata" (Cabré \& Gómez de Enterría, 2006: 23). En definitiva, serían "multidimensionales, multifuncionales y, en consecuencia, poliédricos" (Cabré \& Gómez de Enterria, 2006: 36), por lo que, su caracterización, requeriría una 'aproximación multidimensional'.

Precisamente, Biber y Conrad (2011) proponen un esquema conceptual desgranado en tres niveles -el del 'registro', el del 'género' y el del 'estilo'- que permite una aproximación multifocal a los fenómenos de variación aplicando tres perspectivas concurrentes: "We regard register, genre and style as different approaches or perspectives for analyzing text varieties" (Biber \& Conrad, 2011: 15). Concretamente, en el nivel del 'registro' se estudiarían los rasgos que comparten las producciones verbales de un determinado ámbito funcional que responden a un propósito comunicativo concreto (Biber \& Conrad, 2011: 6): "In general, a register is a variety associated with a particular situation of use (including particular communicative components)". Por su parte, la aproximación desde el 'género' se interesaría por los rasgos tanto funcionales como convencionales de aquellas variedades textuales más representativas de las prácticas sociales de un ámbito comunicativo determinado (Biber \& Conrad, 2011: 16): "they conform to the culturally expected way of constructing texts belonging to the variety". Finalmente, el análisis del 'estilo' permitiría acceder a usos que, en principio, serían puramente convencionales, o, en su caso, responderían a valores meramente estéticos:

"These are features associated with aesthetic preferences, influenced by the attitudes of the speaker/writer about language" (Biber \& Conrad, 2011: 18).

Tal y como evidencian un buen número de trabajos que han abordado la caracterización de textos especializados en diferentes niveles (Biber, 1988, 1995, 2006; Biber \& Finegan, 1994; Conrad \& Biber, 2001; Reppen, Fitzmaurice \& Biber, 2002; Bhatia, Langton \& Lung, 2004; Parodi, 2010; Biber \& Conrad, 2011; etc.), una de las perspectivas que puede resultar más productiva la ofrece la Lingüística del Corpus. De hecho, esta perspectiva ha evidenciado su validez en diversas investigaciones que se han interesado por el uso de marcadores discursivos en textos del ámbito jurídico. Entre ellos podría mencionarse un trabajo de Urrutia (2008) en el que se analizan las 
funciones jurilingüísticas de los marcadores discursivos en la traducción al euskera del Código Civil español; también cabe citar un trabajo de Landone (2010) en el que se estudian los marcadores discursivos más recurrentes en los textos legales de las leyes autonómicas españolas; o un estudio de Mazzi (2011) en el que se analizan los marcadores de reformulación más frecuentes en los textos jurisprudenciales redactados en inglés por dos tribunales europeos.

Por todo ello, en este estudio se parte de un posicionamiento teórico que conjuga, en diferentes niveles, una visión sociocognitiva del discurso, una aproximación a su caracterización desde el variacionismo y la aplicación de los principios de la lingüística de corpus para la recopilación, tratamiento e interpretación de datos. Ello da lugar a un marco teórico relativamente complejo, pero que puede permitir un mejor acercamiento a la 'naturaleza poliédrica' (Cabré \& Enterria, 2006) del objeto de estudio.

\subsection{Categorización de los marcadores del discurso considerados en el estudio}

Para la realización de la toma de datos, en este estudio se han considerado los marcadores discursivos del euskera propuesta por Euskaltzaindia (2008). Se trata de un repertorio inspirado en la categorización propuesta originalmente por Martín Zorraquino y Portolés (1999) para los marcadores del español.

Esta propuesta, basada principalmente en las funciones que desempeñan los marcadores en el discurso, comprende cinco grandes grupos: a) los 'estructuradores de la información' (que sirven, esencialmente, para regular la organización informativa de los discursos, y carecen de significado argumentativo); b) los 'conectores' (marcadores que vinculan semántica y pragmáticamente un miembro del discurso con otro anterior, de tal forma que el marcador guía las inferencias que se han de efectuar del conjunto de los dos miembros discursivos conectados); c) los 'reformuladores' (que presentan el miembro del discurso en el que se encuentran como una expresión más adecuada de lo que se pretendió decir con un miembro precedente); d) los 'operadores argumentativos' (marcadores que por su significado condicionan las posibilidades argumentativas del miembro en el que se incluyen sin relacionarlo con otro anterior); y e) los 'marcadores conversacionales' (las partículas discursivas que aparecen más frecuentemente en la conversación).

La clasificación de Euskaltzaindia (2008) sigue básicamente este esquema, aunque presenta dos pequeñas diferencias con la propuesta original. Por una parte, en el apartado de «conectores〉 se incluye el subgrupo de 〈conectores disyuntivos» no contemplado en la clasificación de marcadores del español; por otra parte, en el quinto grupo se limita a los «marcadores de modalidad epistémica〉. Por lo demás, se limita a distribuir el repertorio de los principales marcadores del euskera según el citado esquema de categorías. 


\section{Diseño y metodología del estudio}

Asumiendo los supuestos que se derivan de los principios de la visión sociocognitiva del discurso, la aproximación variacionista a los usos del lenguaje y las aportaciones de la lingüística del corpus al análisis de dichos usos, la hipótesis sobre la que se asienta el estudio postula que, partiendo de la base de que la selección, frecuencia y concurrencia de los marcadores discursivos puede ser sensible a factores como la naturaleza especializada de los textos, su dominio temático, el registro, el género textual o los criterios estilísticos de los autores, es posible que -dada la particular situación sociolingüística del euskera en el ámbito jurídico- su distribución no sea todo lo consistente y sistemática que pudiera ser deseable. Para evidenciar si los textos de ciencias jurídicas presentan patrones sistemáticos de uso, y para comprobar en qué medida ciertos condicionantes pueden estar provocando distorsiones en dichos patrones, se han analizado tres cuestiones.

En primer lugar, se ha estudiado si las frecuencias de uso de los principales marcadores difieren significativamente en función de dos factores asociados al ámbito discursivo de los textos analizados; concretamente, en función de si se trata o no de textos especializados y, en el caso de los textos de especialidad, en función del dominio temático al que pertenecen (ciencia y tecnología, ciencias sociales y humanas...). En segundo lugar, se ha estudiado si los textos normativos (códigos, leyes generales y autonómicas) y los textos académicos de derecho (monografías, manuales universitarios y artículos doctrinales) presentan distribuciones significativamente diferenciadas en el uso de marcadores. Finalmente, se ha centrado específicamente la atención en los textos normativos traducidos al euskera, y se ha analizado el grado de sistematicidad y convergencia de la distribución de los marcadores discursivos en los textos traducidos por cinco organismos o instituciones: Universidad del País Vasco, Universidad de Deusto, IVAP/IZO, Parlamento Vasco y Parlamento Navarro.

Con este fin, se elaboró un corpus de textos del ámbito de las ciencias jurídicas (Zuzenbide Corpusa) a través de la plataforma HIZLAN ${ }^{5}$, desarrollada precisamente para elaborar, procesar, gestionar, consultar y explotar en línea corpus de esta disciplina ${ }^{6}$. En dicho corpus se recopilaron la totalidad de los textos legislativos publicados en euskera durante el periodo 2000-2011. Además, se incorporaron los manuales universitarios de derecho publicados por la Universidad del País Vasco (UPV/EHU), así como los artículos doctrinales publicados durante la última década en la revista de ciencias jurídicas ELERIA. Además, también se consideró un corpus de trabajos académicos de estudiantes de la Licenciatura de Derecho de la UPV/EHU. Una vez tratados, procesados e indexados, este conjunto de textos acumuló una masa total de 5.735.452 palabras?

Por otra parte, y a a modo de contraste, en la primera fase del estudio se acudió también a diversos corpus de acceso público: EPG (Eredurko Prosa Gaur) ${ }^{8}$ que 
contiene textos periodísticos y literarios; Goenkale Corpusa ${ }^{9}$, que reúne los guiones de la serie televisiva Goenkale, y que, por ello, permite una cierta aproximación a los usos dialógicos de los marcadores; el corpus Pentsamenduaren Klasikoak ${ }^{10}$, que reúne traducciones de obras universales del ámbito de las Ciencias Sociales y de las Humanidades; el corpus $\mathrm{ZIO}^{11}$, en el que se han compilado obras de divulgación científica de reconocido prestigio internacional traducidas al euskera; y ZT corpusa

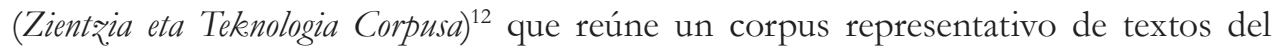
ámbito de las ciencias y de la tecnología publicados en lengua vasca. En total, el conjunto del corpus suma 60.909.969 palabras. Para poder contar con un número suficiente de datos que permitiera operar con pruebas de estadística inferencial, los corpus se segmentaron según el detalle de la Tabla 1.

Tabla 1. Composición y segmentación del corpus de consulta.

\begin{tabular}{|c|c|c|c|c|}
\hline MUESTRA & CORPUS & ÁMBITO & TIPO DOCUMENTOS & $\mathrm{N}^{\circ}$ PALABRAS \\
\hline EPG_PREN & EPG & Periodístico & Noticias & 12.000 .000 \\
\hline EPG_LIB & EPG & Literatura & $\begin{array}{l}\text { Obras literarias contem- } \\
\text { poráneas y ensayos }\end{array}$ & 13.100 .000 \\
\hline GOENKALE & EITB & Interpersonal & Ficción (conversacional) & 1.0982 .625 \\
\hline ZTC_OR & ZTC & Ciencia y tecnología & $\begin{array}{l}\text { Temas científicos interés } \\
\text { general }\end{array}$ & 1.403 .828 \\
\hline ZTC_BZ & ZTC & Ciencia y tecnología & Ciencias de la vida & 2.417 .938 \\
\hline ZTC_LZ & ZTC & Ciencia y tecnología & Ciencias de la tierra & 334.222 \\
\hline ZTC_ME & ZTC & Ciencia y tecnología & Materia y energía & 623.295 \\
\hline ZTC_TE & ZTC & Ciencia y tecnología & Ingeniería y tecnología & 2.083 .004 \\
\hline ZTC_ZZ & ZTC & Ciencia y tecnología & Ciencias exactas & 623.598 \\
\hline ZTC_BS & ZTC & Ciencia y tecnología & Otros & 1.043 .620 \\
\hline $\mathrm{ZIO}$ & $\mathrm{ZIO}$ & Ciencia y tecnología & $\begin{array}{l}\text { Ensayos de divulgación } \\
\text { científica }\end{array}$ & 905.577 \\
\hline KLs_1991-1994 & Klasiko unibertsalak & CCSS y humanidades & $\begin{array}{l}\text { Ensayos (traducidos) (1991- } \\
\text { 1994) }\end{array}$ & 1.640 .625 \\
\hline KLs_1995-1998 & Klasiko unibertsalak & CCSS y humanidades & $\begin{array}{l}\text { Ensayos (traducidos) (1995- } \\
\text { 1998) }\end{array}$ & 2.410 .625 \\
\hline KLs_1999-2006 & Klasiko unibertsalak & CCSS y humanidades & $\begin{array}{l}\text { Ensayos (traducidos) (1998- } \\
\text { 2006) }\end{array}$ & 5.605 .560 \\
\hline GLX_ELB_LT & Zuzenbide Corpusa & Ciencias Jurídicas & Leyes autonómicas (CAPV) & 582.034 \\
\hline GLX_NFL_LT & Zuzenbide Corpusa & Ciencias Jurídicas & Leyes autonómicas (Navarra) & 734.747 \\
\hline GLX_IVAP_IZO_LT & Zuzenbide Corpusa & Ciencias Jurídicas & Leyes generales (IVAP/IZO) & 1.157 .398 \\
\hline GLX_DEUSTU_LT & Zuzenbide Corpusa & Ciencias Jurídicas & $\begin{array}{l}\text { Leyes generales (Universidad } \\
\text { de Deusto) }\end{array}$ & 1.084 .888 \\
\hline GXL_UPV_EHU_LT & Zuzenbide Corpusa & Ciencias Jurídicas & Leyes generales (UPV/EHU) & 203.987 \\
\hline GLX_ELERIA_Art & Zuzenbide Corpusa & Ciencias Jurídicas & $\begin{array}{l}\text { Artículos académicos (ELE- } \\
\mathrm{R} L A)\end{array}$ & 614.221 \\
\hline GLX_UPV_EHU_TL & Zuzenbide Corpusa & Ciencias Jurídicas & $\begin{array}{l}\text { Manuales universitarios } \\
\text { (UPV/EHU) }\end{array}$ & 1.183 .838 \\
\hline GLX_EJ_Art & Zuzenbide Corpusa & Ciencias Jurídicas & $\begin{array}{l}\text { Monografías estudiantes } \\
\text { (UPV/EHU) }\end{array}$ & 174.339 \\
\hline TOTAL & & & & 60.909 .969 \\
\hline
\end{tabular}


Una vez elaborado y segmentado el corpus, se procedió a concretar el repertorio de marcadores a considerar en el estudio. Para ello, se tomó como punto de partida el listado de marcadores compilado en el anteriormente ya citado trabajo de Euskaltzaindia (2008). Dicho listado se amplió sensiblemente incluyendo otros marcadores referenciados en publicaciones sobre cuestiones de estilo y traducción, que, aun siendo de uso poco frecuente en el lenguaje común, presumiblemente podrían ser de especial interés en los textos del ámbito jurídico. De ello resultó el listado de la Tabla 2. En dicha Tabla se presentan los marcadores analizados, distribuidos por categorías según el esquema de Euskaltzaindia (2008), al que se ha hecho anteriormente referencia en el epígrafe 1.5.

Tabla 2. Listado de partículas analizadas.

\begin{tabular}{|c|c|c|}
\hline Grupo & Categoría & Marcadores analizados \\
\hline \multirow[t]{3}{*}{$\begin{array}{l}\text { 1. Estructuradores } \\
\text { de la información }\end{array}$} & 1.1. Comentadores & $\begin{array}{l}\text { gauzak horrela, horiek horrela, ildo horretatik, betiere, ezen, } \\
\text { izan ezik, izan ezean, salbu, segun eta, non eta, noiz eta, baldin } \\
\text { eta, harik eta }\end{array}$ \\
\hline & 1.2. Ordenadores & $\begin{array}{l}\text { lebenik, eta bebin, lehenik, bigarrenik, birugarrenik, azkenik, } \\
\text { batetik, bestetik, alde batetik, beste alde batetik, basteko, } \\
\text { jarraitzeko, amaitzeko, basieran, ondoren, jarraian, bitartean, } \\
\text { amaieran }\end{array}$ \\
\hline & 1.3. Digresores & hori dela eta, bide batez, bidenabar \\
\hline \multirow[t]{2}{*}{$\begin{array}{l}\text { 2. Operadores } \\
\text { discursivos }\end{array}$} & 2.1. De refuerzo & $\begin{array}{l}\text { benetan, zinez, berez, ezinbestean, egiazki, bereziki, nagusiki, } \\
\text { gehienbat, batez ere }\end{array}$ \\
\hline & 2.2. De concreción & $\begin{array}{l}\text { adibidez, esaterako, esate baterako, konparaziorako, kasu, bala } \\
\text { nola, besteak beste, hain zuzen, zehazki, burrenez hurren }\end{array}$ \\
\hline \multirow[t]{5}{*}{ 3. Conectores } & 3.1. Aditivos & $\begin{array}{l}\text { eta, baita, ezta, ere, ez ezik, halaber, orobat, berebat, gainera, } \\
\text { horrez gain, hortik landa, borrez, aparte, bestalde, behintzat, } \\
\text { behinik behin, bederen, bederik, badere, baderik. }\end{array}$ \\
\hline & 3.2. Disyuntivos & $\begin{array}{l}\text { edo, ala, edota, nabiz, zein, bestela, osterantzean, gainerantz- } \\
\text { ean, ezpere(n), ezpabere }\end{array}$ \\
\hline & $\begin{array}{l}\text { 3.3. Contraargumen- } \\
\text { tativos }\end{array}$ & $\begin{array}{l}\text { baina, ordea, aldiz, berriz, ostera, aitzitik, alta, alabaina, ala- } \\
\text { badere, dena dela, dena den, bala ere, hargatik, haatik, barren, } \\
\text { badarike ere, baizik, baizik, eta, }\end{array}$ \\
\hline & 3.4. Consecutivos & beraz, hortaz, ondorioz, honenbestez, halatan, hala, bada \\
\hline & 3.5. Causales & zeren, izan ere \\
\hline \multirow[t]{4}{*}{ 4. Reformuladores } & 4.1. Explicativos & bau da, hots, alegia \\
\hline & 4.2. De rectificación & bobe(to) esanda, bestela esanda, beste bitz batzuetan \\
\hline & 4.3. De distanciamiento & $\begin{array}{l}\text { zernahi gisaz, nolanabi ere, edozelan ere, edozein modutan ere, } \\
\text { edozein moduz, guztiarekin ere }\end{array}$ \\
\hline & 4.4. Recapitulativos & $\begin{array}{l}\text { laburbilduz(ta)(ik), labur esan(da), bitz gutxitan, arken } \\
\text { batean, aqken finean, azken buruan }\end{array}$ \\
\hline $\begin{array}{l}\text { 5. Marcadores } \\
\text { epistémicos }\end{array}$ & 5.1. Epistémicos & omen, ote, noski, jakina, dirudienez, antra denez. \\
\hline
\end{tabular}

La recogida de datos se realizó a través de la interfaz de consulta que ofrecen los corpus considerados en el estudio. No obstante, los resultados fueron cribados manualmente para descartar posibles errores (producto de deficiencias de lematización 
o etiquetado) o casos en los que la partícula en cuestión no desempeñara la función de marcador discursivo. Los resultados se recogieren en una base de datos, la cual acumuló cerca de 30.000 registros.

Estos datos fueron sometidos a diversas pruebas estadísticas con el programa IBM SPSS Statistics 19. Para determinar si los datos sugieren la existencia de correlación significativa entre las frecuencias de aparición de los marcadores y las variables del estudio (el carácter especializado de los textos, el dominio de especialidad, el registro, el género textual y la autoría), se aplicó la prueba de correlación de Pearson. Para confirmar que las distribuciones de los marcadores agrupados en cada una de las categorías presentaban distribuciones consistentes en la totalidad de las muestras se aplicó un análisis ANOVA de una sola variable para cada una de ellas. Finalmente, para poder determinar qué marcadores discursivos presentan patrones de distribución correlacionados entre sí y cuáles son los marcadores que determinan la idiosincrasia de los diversos grupos de producciones analizados, se realizó un Análisis Factorial de Componentes Principales. El conjunto de datos y gráficos se archivaron en formato digital, y las tablas de resultados se recopilaron y analizaron en un informe de investigación no publicado (Ezeiza, 2011b) ${ }^{13}$.

\section{Resultados y discusión}

Siguiendo el esquema propuesto para el estudio, en primer lugar, analizó si los datos de frecuencia de uso de los marcadores discursivos sugieren algún tipo de correlación entre la dicha distribución y la naturaleza especializada (o no) de los textos y, en su caso, el ámbito de temático al que dichos textos pertenecen (factores que, para simplificar la presentación de resultados se han agrupado bajo la denominación 'ámbito discursivo'). Con este fin, los datos de frecuencias de uso de los marcadores detectados en los diferentes grupos de textos considerados (ficción televisiva, prensa, literatura, ciencia, tecnología, ciencias sociales, humanidades y ciencias jurídicas) fueron sometidos a las pruebas de correlación y de regresión previstas en el diseño metodológico. Los resultados fueron claramente concluyentes. De hecho, tal y como se puede apreciar en la Tabla 3, los índices de correlación resultaron ser superiores a 0,80 para todas las categorías de marcadores. Además, casi todos los grupos de marcadores arrojaron en la prueba ANOVA índices de regresión significativos, lo cual indicaría que, en conjunto, los diferentes marcadores pertenecientes a cada categoría presentan distribuciones consistentes en los diferentes grupos de textos considerados. De ello, cabe deducir que las diferencias observadas en función de la naturaleza especializada (o no) de los textos y de su ámbito temático podrían no ser arbitrarias, y que, por otra parte, las frecuencias de uso de los marcadores observadas en los textos jurídicos podrían considerarse distintivas del discurso de dicho ámbito. 
Tabla 3. Resultados correspondientes a la variable ‘ámbito discursivo'.

\begin{tabular}{|c|c|c|c|c|c|}
\hline \multicolumn{2}{|l|}{ MARCADORES } & \multicolumn{2}{|l|}{ Ajuste de la distribución } & \multicolumn{2}{|c|}{$\begin{array}{l}\text { ANOVA de una } \\
\text { variable }\end{array}$} \\
\hline GRUPO & CATEGORÍA & ECUACIÓN DE AJUSTE & $\mathbf{R}^{2}$ & $\mathbf{R}^{2}$ & Sig. (4) \\
\hline \multirow{5}{*}{$\begin{array}{l}\text { 1. Estructuradores de la } \\
\text { información }\end{array}$} & 1.1. Comentadores & $\mathrm{y}=1,6727 \mathrm{e}^{0,1651 \mathrm{x}}$ & 0,8857 & 0,943 & $0,001^{* *}$ \\
\hline & 1.2. Ordenadores-1 & \multirow{3}{*}{$y=1,6727 e^{0,1651 x}$} & \multirow{3}{*}{0,8685} & 0,918 & $0,020^{*}$ \\
\hline & 1.2. Ordenadores- 2 & & & 0,596 & $0,001^{* *}$ \\
\hline & 1.2. Ordenadores-3 & & & 0,698 & $0,030^{*}$ \\
\hline & 1.3. Digresores & $y=0,3567 e^{0,0902 x}$ & 0,8321 & 0,688 & $0,008^{* *}$ \\
\hline \multirow{3}{*}{$\begin{array}{l}\text { 2. Operadores discur- } \\
\text { sivos }\end{array}$} & 2.1. De refuerzo-1 & \multirow{2}{*}{$y=7,2287 e^{0,0524 x}$} & \multirow{2}{*}{0,8167} & 0,660 & 0,179 \\
\hline & 2.1. De refuerzo-2 & & & 0,428 & $0.040 *$ \\
\hline & 2.2. De concreción & $y=1,0934 X+3,319$ & 0,8626 & 0,788 & $0,015^{* *}$ \\
\hline \multirow{9}{*}{ 3. Conectores } & 3.1. Aditivos & $\mathrm{y}=85,746 \mathrm{e}^{0,1098 \mathrm{x}}$ & 0,7850 & 0,984 & $0.040^{*}$ \\
\hline & 3.2. Disyuntivos & $y=31,823 e^{0,0855 x}$ & 0,8638 & 0,779 & $0,018^{* *}$ \\
\hline & $\begin{array}{l}\text { 3.3. Contraargumentati- } \\
\text { vos-1 }\end{array}$ & \multirow{2}{*}{$y=17,969 e^{0,0558 x}$} & \multirow{2}{*}{0,8462} & 0,836 & 0,564 \\
\hline & $\begin{array}{l}\text { 3.3. Contraargumentati- } \\
\text { vos-2 }\end{array}$ & & & 0,507 & $0,031 *$ \\
\hline & 3.4. Consecutivos-1 & \multirow{2}{*}{$y=4,6683 X^{0,6786}$} & \multirow{2}{*}{0,9001} & 0,369 & 0,379 \\
\hline & 3.4. Consecutivos- 2 & & & 0,652 & $0,05^{*}$ \\
\hline & 3.5. Causales-1 & \multirow{3}{*}{$y=1,2091 e^{0,1459 x}$} & \multirow{3}{*}{0,9208} & 0,239 & 0,074 \\
\hline & 3.5. Causales-2 & & & 0,497 & $0,004^{* *}$ \\
\hline & 3.5. Causales-3 & & & 0,488 & $0,009^{* *}$ \\
\hline \multirow{5}{*}{ 4. Reformuladores } & 4.1. Explicativos & $y=1,3164 x-2,1668$ & 0,9089 & 0,172 & 0,323 \\
\hline & 4.2. De rectificación & $\begin{array}{l}y=0,0019 X^{2}+0,0178 X- \\
0,0034\end{array}$ & 0,8030 & 0,525 & $0,003^{* *}$ \\
\hline & 4.3. De distanciamiento & $y=0,0098 X^{2}-0,075 X+0,9923$ & 0,8137 & 0,551 & $0,03^{*}$ \\
\hline & 4.4. Recapitulativos-1 & \multirow{2}{*}{$y=0,3458 e^{0,0935 x}$} & \multirow{2}{*}{0,9068} & 0,449 & 0,123 \\
\hline & 4.4. Recapitulativos-2 & & & 0,412 & $0,05^{*}$ \\
\hline $\begin{array}{l}\text { 5. Marcadores epis- } \\
\text { témicos }\end{array}$ & 5.1. Epistémicos & $y=0,8664 e^{0,1637 x}$ & 0,8140 & 0,611 & $0,015^{* *}$ \\
\hline
\end{tabular}

De hecho, tal y como se observa en el ejemplo del Gráfico 1, las representaciones de las líneas de ajuste correspondientes a las distribuciones de frecuencias de uso de los marcadores evidencian, que los textos de cada uno de los ámbitos considerados (prensa, literatura, ciencia y tecnología, ciencias sociales y humanas, y ámbito jurídico) tienden a agruparse en segmentos diferenciados de la línea de distribución. Esto es, coherente con la hipótesis de que el carácter especializado (o no) de los textos, y el ámbito temático al que pertenecen, puedan tener su reflejo en la selección y uso de marcadores. 


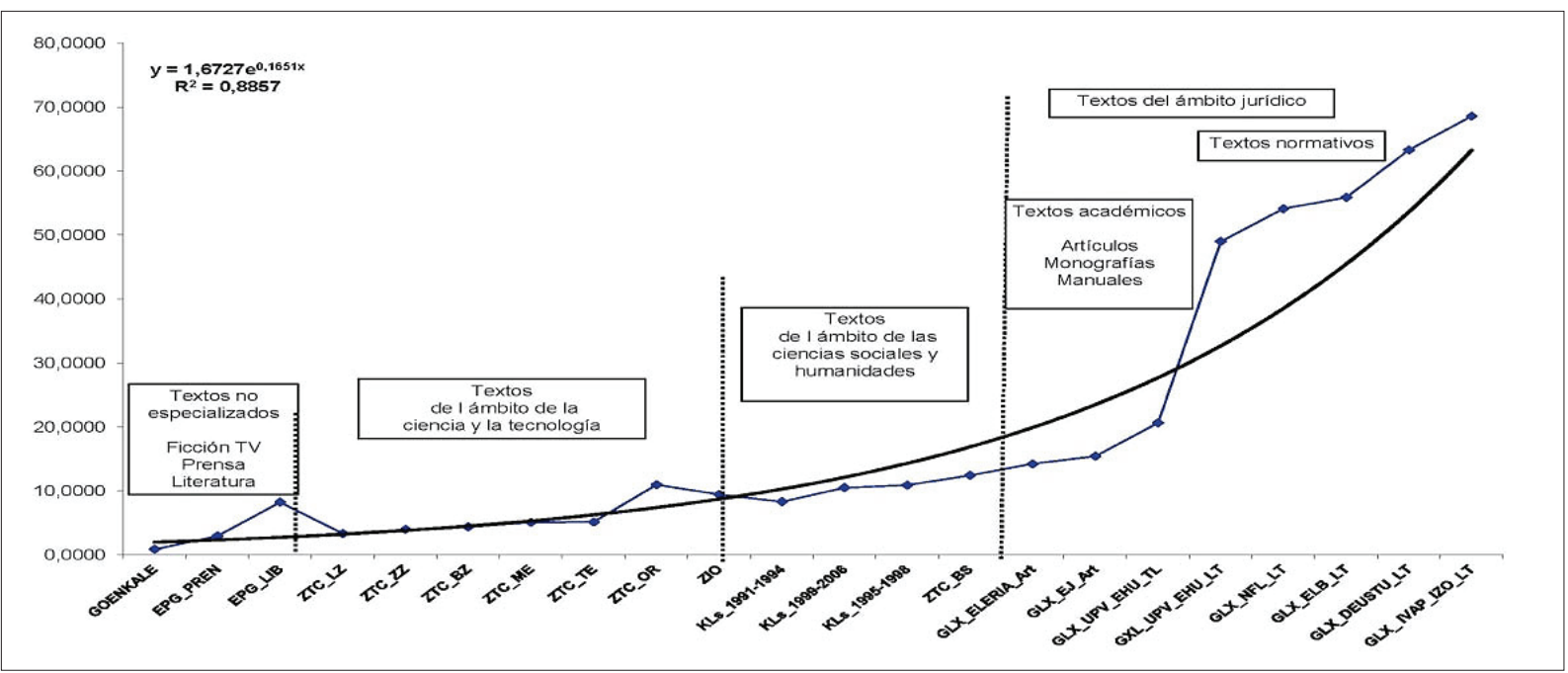

Gráfico 1. Línea de distribución de frecuencias de marcadores de la categoría 1.1 en función del 'ámbito discursivo'.

Igualmente, en el segmento correspondiente a los textos jurídicos se observa un punto de inflexión que establecería el límite entre textos académicos y textos normativos. Las pruebas estadísticas realizadas (algunos de cuyos resultados se recogen en la Tabla 3), dejaron patente que las diferencias que dan lugar a dicha inflexión serían indicativas de patrones diferenciados, lo cual parece sugerir que, en los textos jurídicos, las frecuencias de uso de marcadores podrían diferir en el registro normativo y en el registro académico.

A la vista de estos resultados, a continuación se trató de clarificar si los patrones de distribución pudieran ser sensibles a la variable 'género textual'. Para ello, se segregaron los datos de frecuencia detectados en los textos normativos (considerando de forma independiente los correspondientes a códigos, leyes generales y leyes autonómicas) y en los de los textos académicos (considerando de forma independiente los correspondientes a los artículos doctrinales, manuales universitarios y monografías). Dichos datos fueron sometidos a las pruebas de correlación y de regresión previstas en el diseño del estudio. Tal y como se puede apreciar en las columnas tercera y quinta de la Tabla 4, dichas pruebas arrojaron resultados significativos para prácticamente todos los grupos de marcadores. Por lo tanto, cabe deducir que las diferencias detectadas en las frecuencias de uso de los marcadores parecen responder a patrones de variación sistemáticos, y que, en definitiva, permiten postular que los diversos grupos textuales analizados presentan configuraciones dependientes del 'género' al que pertenece cada uno de ellos. 
Tabla 4. Resultados correspondientes a las variables 'género textual' y 'autoría'.

\begin{tabular}{|c|c|c|c|c|c|}
\hline \multirow{3}{*}{ GRUPO } & \multirow{3}{*}{ CATEGORÍA } & \multirow{2}{*}{\multicolumn{2}{|c|}{$\begin{array}{c}\text { Prueba de correlación de } \\
\text { Pearson }\left(\mathbf{R}^{2}\right)\end{array}$}} & \multirow{2}{*}{\multicolumn{2}{|c|}{$\begin{array}{c}\text { ANOVA de una variable }\left(\mathbf{R}^{2}\right) \\
\text { VARIABLES }\end{array}$}} \\
\hline & & & & & \\
\hline & & GÉNERO & AUTORÍA & GÉNERO & AUTORÍA \\
\hline \multirow{3}{*}{$\begin{array}{l}\text { 1. Estructuradores de la } \\
\text { información }\end{array}$} & 1.1. Comentadores & $-0,830 * *$ & $-0,905 * *$ & $0,897 * *$ & $0,886^{* *}$ \\
\hline & 1.2. Ordenadores & $0,887 * *$ & $0,758 * *$ & $0,956 * *$ & $0,932 * *$ \\
\hline & 1.3. Digresores & $0,534 * *$ & $0,527 * *$ & $0,897 * *$ & $0,366 * *$ \\
\hline \multirow[t]{2}{*}{ 2. Operadores discursivos } & 2.1. De refuerzo & $0,391 * *$ & $0,476 * *$ & $0,627 * *$ & $0,589 * *$ \\
\hline & 2.2. De concreción & $0,836 * *$ & $0,863 * *$ & $0,885 * *$ & $0,900 * *$ \\
\hline \multirow[t]{5}{*}{ 3. Conectores } & 3.1. Aditivos & $-0,465 * *$ & $-0,416$ & $0,957 * *$ & $0,893 * *$ \\
\hline & 3.2. Disyuntivos & $-0,694 * *$ & $-0,811 * *$ & $0,699 * *$ & $0,751 * *$ \\
\hline & 3.3. Contraargumentativos & $0,835 * *$ & $0,779 * *$ & $0,958 * *$ & $0,892 * *$ \\
\hline & 3.4. Consecutivos & $0,777 * *$ & $0,582 * *$ & $0,853 * *$ & $0,847 * *$ \\
\hline & 3.5. Causales & $0,845 * *$ & $0,822 * *$ & $0,769 * *$ & $0,702 * *$ \\
\hline \multirow[t]{4}{*}{ 4. Reformuladores } & 4.1. Explicativos & $0,775 * *$ & $0,831 * *$ & $0,628 * *$ & $0,709 * *$ \\
\hline & 4.2. De rectificación & $0,558 * *$ & $0,505^{* *}$ & $0,371 *$ & $0,342 *$ \\
\hline & 4.3. De distanciamiento & 0,344 & 0,069 & 0,470 ** & $0,535 * *$ \\
\hline & 4.4. Recapitulativos & $0,660 * *$ & $0,608 * *$ & $0,783 * *$ & $0,747 * *$ \\
\hline 5. Epistémicos & 5.1. Epistémicos & $0,810 * *$ & $0,782 * *$ & $0,800 * *$ & $0,662 * *$ \\
\hline
\end{tabular}

En cuarto lugar, se procedió a analizar la posible influencia de la autoría en ciertas diferencias sensibles que se observaron en los textos normativos traducidos por diferentes instituciones. Para ello se segregaron los datos correspondientes a los textos traducidos por el Parlamento Vasco, el Parlamento Navarro, el Servicio Oficial de Traducción del IVAP, la Universidad de Deusto y la Universidad del País Vasco, y, al igual que en las anteriores fases del estudio, fueron sometidos a las correspondientes pruebas de correlación y de regresión. Tal y como se puede comprobar en los datos de las columnas cuatro y seis de la Tabla 4, las pruebas de correlación y regresión sugieren una presumible influencia de la autoría en las frecuencias de uso en el caso de la mayor parte de grupos de marcadores considerados. Este resultado no es especialmente sorprendente, si se tiene en cuenta que hasta fechas recientes no han comenzado a dar frutos los esfuerzos por aunar criterios para consolidar un modelo de lenguaje legislativo común en lengua vasca. No obstante, las diferencias observadas entre textos de diferentes autores son más leves y sutiles que las observadas entre los textos legislativos y los académicos. Por todo ello, cabe postular que las diferencias detectadas en los patrones de uso se podrían deber a criterios de 'estilo'.

A la luz de estos resultados, los textos del ámbito jurídico presentarían frecuencias de uso de marcadores claramente distintivas e idiosincrásicas, y dentro de los textos de este ámbito se observarían unos patrones de distribución complejos, los cuales se evidenciarían en las tres dimensiones del modelo de Biber y Conrad (2011). A la vista de todo ello, y comoquiera que en las fases anteriores los cálculos se realizaron de forma independiente para cada grupo de marcadores, en la última parte del estudio se trató de dilucidar si en los patrones de variación pudieran existir concurrencias 
complejas entre marcadores de diferentes categorías, y si dichas concurrencias pudieran atribuirse al 'registro', al 'género' o al 'estilo' de los textos considerados. Para aclarar esta cuestión, el conjunto de datos fue sometido a una operación de reducción de variables mediante un Análisis Factorial de Componentes Principales. En la Tabla 5 se presentan los resultados correspondientes a los tres principales factores, que en conjunto explicarían el 79,39\% de la varianza, y que, por lo tanto, pueden ser considerados suficientemente representativos de la variabilidad detectada en los textos analizados. En cada una de las columnas se destacan en negrita los coeficientes que indican cuáles son los componentes con mayor influencia en cada factor.

Tabla 5. Resultados de Análisis Factorial de Componentes Principales.

\begin{tabular}{|l|c|c|c|}
\cline { 2 - 4 } \multicolumn{1}{c|}{} & \multicolumn{3}{c|}{ Matriz de componentes } \\
\cline { 2 - 4 } \multicolumn{1}{c|}{} & Factor 1 & Factor 2 & Factor 3 \\
\hline 1.1. Comentadores & $-0,624$ & $\mathbf{- 0 , 6 4 4}$ & $-0,238$ \\
\hline 1.2. Ordenadores & $\mathbf{0 , 7 0 8}$ & 0,334 & 0,467 \\
\hline 1.3. Digresores & $\mathbf{0 , 9 3 1}$ & 0,036 & $-0,115$ \\
\hline 2.1. De refuerzo & 0,179 & $\mathbf{0 , 7 6 5}$ & $-0,375$ \\
\hline 2.2. De concreción & 0,581 & $\mathbf{0 , 6 5 3}$ & 0,319 \\
\hline 3.1. Aditivos & $-0,134$ & 0,116 & $-0,915$ \\
\hline 3.2. Disyuntivos & $-0,541$ & $\mathbf{- 0 , 7 1 0}$ & $-0,018$ \\
\hline 3.3. Contraargumentativos & $\mathbf{0 , 6 9 1}$ & 0,408 & 0,497 \\
\hline 3.4. Consecutivos & $\mathbf{0 , 6 6 0}$ & 0,154 & 0,616 \\
\hline 3.5. Causales & $\mathbf{0 , 8 1 0}$ & 0,386 & 0,297 \\
\hline 4.1. Explicativos & $\mathbf{0 , 6 3 1}$ & 0,530 & 0,482 \\
\hline 4.2. De rectificación & $\mathbf{0 , 7 8 5}$ & 0,030 & 0,278 \\
\hline 4.3. De distanciamiento & $-0,221$ & $\mathbf{0 , 5 2 6}$ & $-0,057$ \\
\hline 4.4. Recapitulativos & 0,418 & $\mathbf{0 , 5 9 4}$ & 0,285 \\
\hline 5.1. Epistémicos & 0,321 & $\mathbf{0 , 7 5 2}$ & 0,460 \\
\hline
\end{tabular}

\begin{tabular}{|c|c|c|}
\hline \multicolumn{3}{|c|}{$\begin{array}{c}\text { Matriz de coeficientes para el cálculo de } \\
\text { las puntuaciones en las componentes }\end{array}$} \\
\hline Factor 1 & Factor 2 & Factor 3 \\
\hline$-0,053$ & $-0,133$ & 0,023 \\
\hline 0,105 & $-0,026$ & 0,091 \\
\hline 0,455 & $-0,227$ & $-0,343$ \\
\hline$-0,007$ & 0,295 & $-0,275$ \\
\hline 0,006 & 0,150 & 0,041 \\
\hline 0,174 & 0,074 & $-0,532$ \\
\hline$-0,061$ & $-0,181$ & 0,136 \\
\hline 0,070 & 0,013 & 0,116 \\
\hline 0,087 & $-0,092$ & 0,196 \\
\hline 0,189 & $-0,031$ & $-0,044$ \\
\hline 0,014 & 0,084 & 0,126 \\
\hline 0,275 & $-0,186$ & $-0,054$ \\
\hline$-0,223$ & 0,295 & 0,037 \\
\hline$-0,043$ & 0,165 & 0,066 \\
\hline$-0,181$ & 0,265 & 0,206 \\
\hline
\end{tabular}

A la vista de estos resultados, se puede concluir que la distribución general de marcadores discursivos se articularía principalmente en torno a dos ejes de variación.

1. El eje principal de variación implicaría siete grupos de marcadores: ordenadores, digresores, contraargumentativos, consecutivos, causales, explicativos y de rectificación.

2. El segundo eje de variación implicaría otros seis grupos de marcadores: los de refuerzo, los de concreción, los disyuntivos, los de distanciamiento, los recapitulativos y los epistémicos.

Los datos son bastante rotundos (el coeficiente más bajo tendría un valor de $0,526)$, lo cual implica que la mayor parte de los grupos de marcadores varían de forma sistemática y concurrente en torno a los dos ejes principales.

El primero de los factores parece representar un eje de variación que se podría asociar a tres funciones de la marcación en el discurso: a) la de regulación de la 
información en el texto (vinculada al uso de estructuradores de la información, fundamentalmente de las categorías 1.2 y 1.3 , pero también de los estructuradores de la categoría 1.1); b) las operaciones de explicación y rectificación (evidenciadas mediante al uso de reformuladores de las categorías 4.1 y 4.2); y c) la realización de instrucciones inferenciales de causa, consecuencia, contraargumentación y, parcialmente, de disyunción (asociadas al uso de conectores de las categorías 3.2, 3.3, 3.4 y 3.5$)$.

El segundo de los factores, por su parte, parece representar un eje de variación que se podría asociar a las siguientes funciones: a) las de condicionamiento argumentativo de concreción y de refuerzo (evidenciadas mediante el uso de marcadores de las categorías 2.1 y 2.2); b) las operaciones de distanciamiento y recapitulación (vinculadas al uso de reformuladores de las categorías 4.3 y 4.4); y c) la explicitación de la actitud (marcada mediante el uso de epistémicos). En otras palabras, los resultados del análisis factorial señalan unos patrones de covariación de diversas categorías de marcadores que, en conjunto, parecen estar relacionados con la marcación de dos grandes grupos de estrategias: i) unas estarían fundamentalmente orientadas a gestionar la información en el texto a través de operaciones de ordenación, explicación, causa, consecuencia, digresión, rectificación, etc.; y ii) las otras estarían más enfocadas a orientar la interpretación discurso mediante operaciones de concreción, refuerzo, posicionamiento, distanciamiento o recapitulación.

Para observar en qué grado esta aproximación matemática revela un comportamiento o una distribución que pueda considerarse consistente -esto es, para determinar en qué grado las concurrencias y correlaciones detectadas parecen responder a una cierta coherencia lingüística-, las puntuaciones factoriales fueron representadas gráficamente en varios mapas de distribución con diferente nivel de detalle (ver Gráficos 2, 3 y 4).

Tal y como se puede apreciar, en el Gráfico 2 las muestras correspondientes a los textos normativos se enmarcan en un sector muy acotado del cuadrante inferior izquierdo del mapa factorial. Por lo tanto, se caracterizarían por una frecuencia moderada y equilibrada del conjunto de marcadores idiosincrásicos de los textos de este ámbito. La distribución de las muestras correspondientes a los textos académicos presenta, sin embargo, una mayor dispersión. Algunos de estos grupos de textos se caracterizarían por una alta frecuencia de marcadores asociados al primer eje de variación y una baja frecuencia de marcadores asociados al segundo de los ejes. Otros presentarían la configuración inversa. De ello parece deducirse que en el registro normativo el uso de marcadores es bastante más pautado y regular que en el registro académico. No obstante, tal y como deja patente el Gráfico 3, la dispersión observada en los textos académicos no sería arbitraria, ya que si se diferencian los puntos correspondientes a los artículos doctrinales, los manuales universitarios, y a las monografías del alumnado, se observan dos patrones claramente diferenciados (los correspondientes a los artículos y a los manuales), y un patrón híbrido (el de las 
monografías de los estudiantes), cuya configuración cabalgaría entre la del discurso que reciben como input (el de los manuales) y el discurso que deben ir adquiriendo (el de los expertos). Así pues, todo ello parece sugerir un cierto vínculo entre el uso de marcadores y el 'género textual' de las variedades de textos consideradas en el estudio.

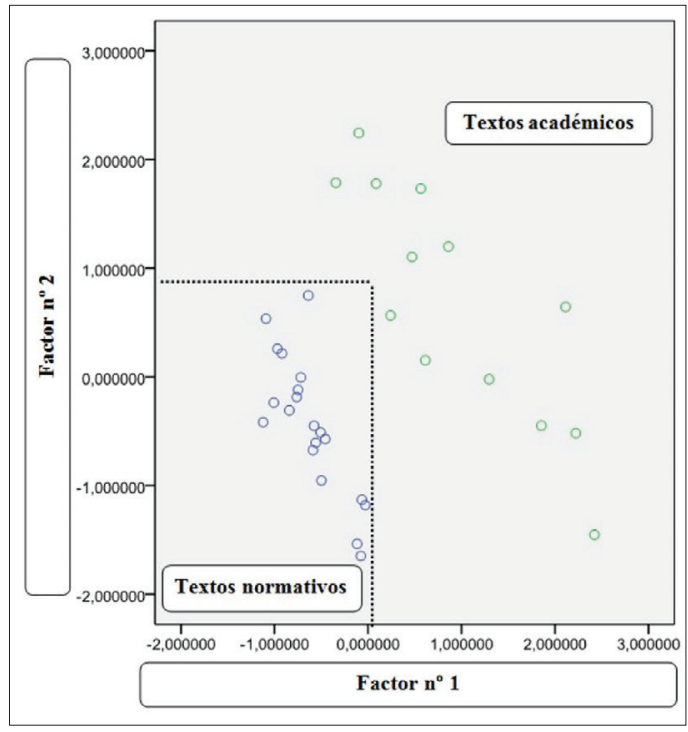

Gráfico 2. Distribución factorial (I): 'registro'.

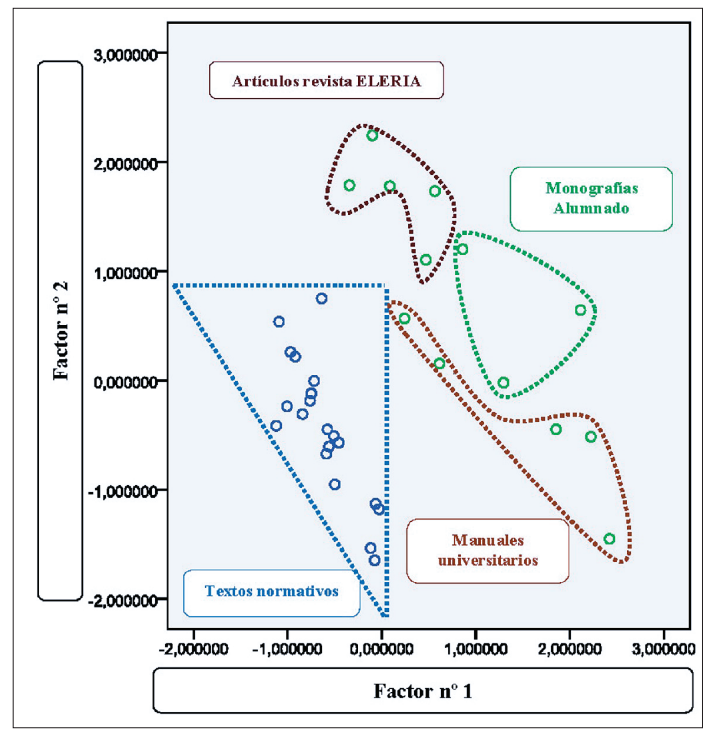

Gráfico 3. Distribución factorial (II): 'género textual'.

Por otra parte, si se aíslan los datos correspondientes a los textos normativos, se puede obtener una representación gráfica con mayor nivel de detalle, en la que se comprueba que la dispersión observada en el sector ocupado por estos textos dentro del mapa factorial, tampoco parece obedecer al azar. De hecho, tal y como se puede observar en el Gráfico 4, en la parte inferior se agrupan las leyes generales traducidas por la Universidad de Deusto, y por el Servicio Oficial de Traducción del Instituto Vasco de Administración Pública del Gobierno Vasco (IVAP/IZO). Esta concurrencia no es sorprendente dado que ambas instituciones llevan al menos dos décadas trabajando conjuntamente en la traducción de textos legales. Por otra parte, en el extremo opuesto del mapa factorial se sitúan los textos legislativos del Parlamento Navarro y del Parlamento Vasco. Ello podría explicarse por las diferencias entre textos legislativos generales (más extensos, principalmente dirigidos a operadores jurídicos, influidos por cierta tradición histórica...) y los textos normativos autonómicos (breves, dirigidos a empresas y ciudadanía, de temática concreta y acotada...). Finalmente, en la posición media se situarían los textos legislativos traducidos por la Universidad del País Vasco (UPV/EHU). Esto tampoco resulta sorprendente, ya que el proyecto Legeak/Leyes de traducción legislativa nació, precisamente, con la vocación de facilitar la convergencia de los diferentes modelos de prosa jurídica existentes, con una propuesta unificadora. 


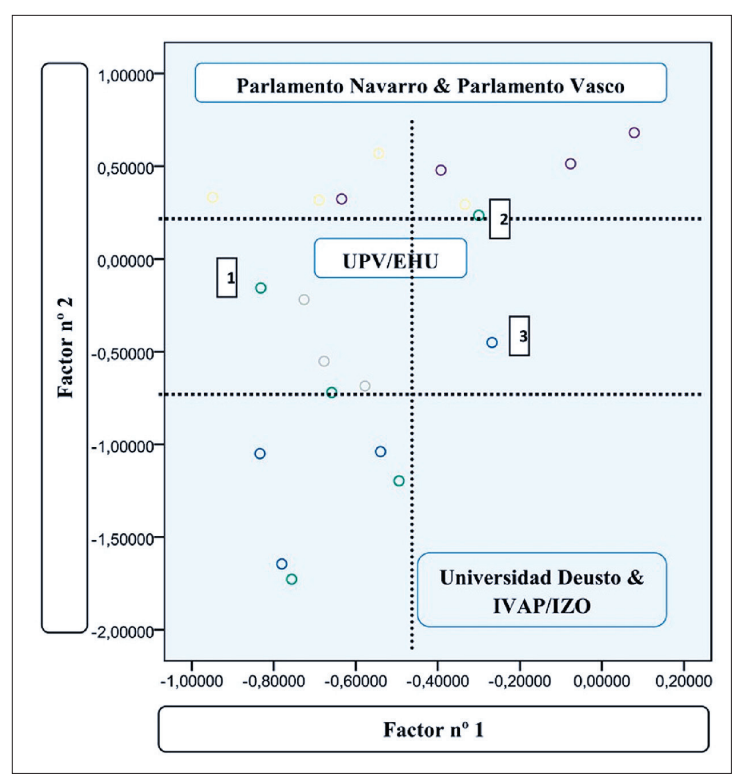

Gráfico 4. Distribución factorial (III): 'autoría' / 'estilo'.

Pero probablemente el hallazgo más revelador lo ofrecen las muestras señaladas en el Gráfico 4 con los números 1, 2 y 3 . Se trata de tres grupos de textos que, en principio, aparentan estar desubicados, ya que se trata de grupos de textos normativos traducidos por la Universidad de Deusto (1 y 2) o el Servicio Oficial de Traducción (3). Por lo tanto, cabría esperar que se situaran en la parte baja de la distribución.

Sin embargo, se sitúan en la órbita de los textos de la UPV/EHU. De hecho, se trata de textos publicados entre los años 2005 y 2007. Por lo tanto, habrían sido traducidos con posterioridad a varios simposios e iniciativas desarrolladas en el ámbito del euskera jurídico con el fin de explorar vías de convergencia entre los modelos de prosa jurídica existentes. Esta disposición en el mapa factorial parece sugerir la emergencia de una configuración estilística compartida y común en vías de consolidación.

Como se puede apreciar, los resultados del estudio resultan reveladores en varios sentidos. Por una parte, desde la perspectiva jurilingüística, los resultados resultan relativamente tranquilizadores, ya que parecen señalar que la variación detectada en el uso de marcadores es altamente sistemática y consistente, de forma particular en los textos más recientes. Por otra parte, las pruebas de análisis estadístico permiten identificar cuáles son los grupos de marcadores con mayor trascendencia en las variedades textuales analizadas.

Se trataría de los dos grandes grupos de marcadores en torno a los que pivotan los principales ejes de variación. Al respecto, cabe apuntar que los resultados obtenidos coinciden en gran medida con los de Landone (2010). Esta coincidencia de resultados 
parece aportar cierta validez al trabajo aquí presentado, dado que parece ratificar (aun en perspectiva translingüística) la sospecha de Landone (2010) de que los textos normativos autonómicos presentan ciertas peculiaridades idiosincrásicas en el uso de marcadores.

Con respecto al trabajo de Mazzi (2011), no parece que nuestro estudio pueda ser de gran utilidad para corroborar o refutar sus resultados. En él se analiza la función desambiguadora de los marcadores de reformulación en sentencias judiciales. No obstante, y aun cuando en nuestro trabajo no se han considerado textos judiciales, cabe señalar que en los textos normativos estudiados los marcadores de reformulación cumplen relevantes funciones jurilingüísticas. De hecho, un trabajo reciente (Alberdi \& Ezeiza, 2013) ratifica este extremo, particularmente desde la perspectiva de las estrategias de traducción.

Finalmente, debemos referirnos necesariamente al trabajo de Urrutia (2008), dado que es el que precisamente inspiró nuestro estudio. Como ya se ha comentado anteriormente, en él se ofrece una visión general del repertorio de marcadores utilizados en la traducción al euskera del Código Civil español, siguiendo el esquema taxonómico propuesto en el trabajo de Euskaltzaindia (2008). La finalidad de su trabajo no era otra que llamar la atención sobre la relevancia que puede tener para el desarrollo del euskera jurídico ampliar el foco de interés a aspectos relacionados con el discurso, y particularmente, al del uso de los marcadores discursivos. Pues bien, creemos que los datos ofrecidos en este trabajo evidencian la validez de su tesis, y que invitan a seguir estudiando el funcionamiento de los marcadores desde diversas perspectivas. Los resultados obtenidos - en este caso mediante metodología meramente estadística-apuntan ya una serie de prioridades y algunos criterios que pueden resultar de utilidad para elaborar una agenda de investigación, y para clarificar, aun provisionalmente, algunas dudas que nos asisten sobre la cuestión.

\section{CONCLUSIONES}

De acuerdo con lo expuesto en el apartado 3, los resultados arrojados por el estudio han permitido dar respuesta, al menos parcialmente, a las preguntas de investigación planteadas. Por una parte, los resultados sugieren que la frecuencia, distribución y concurrencia de los marcadores discursivos podría estar influida por la naturaleza especializada o no de los textos y, en el caso de los textos especializados, por el dominio temático al que pertenecen. Por otra parte, dentro del ámbito de las ciencias jurídicas se ha observado la existencia de diferentes patrones de distribución, cuya sistematicidad sugiere que la variación observada sería coherente con lo postulado por Loureda y Acín (2010) con respecto la naturaleza discursivo-pragmática de los marcadores. De ello cabe deducir, varias conclusiones.

En primer lugar, los resultados parecen corroborar la tesis de los trabajos de Euskaltzaindia (2008) y Urrutia (2008), según la cual los marcadores 
discursivos constituyen un recurso lingüístico 'estratégico' no solo para una prosa comunicativamente eficaz; además, los marcadores discursivos tendrían también una relevancia particularmente destacada en la prosa jurídica. De hecho, aun cuando se cuente con solo contados trabajos sobre la cuestión (Landone, 2010; Mazzi, 2011; Alberdi \& Ezeiza, 2013; Ezeiza, 2014), todos ellos parecen apoyar esta tesis. Ello hace pensar que esta vía de investigación puede resultar de especial interés aplicado, particularmente desde la perspectiva del 'derecho a comprender', que requiere formas de expresión acordes con el criterio de seguridad jurídica. Por otra parte, el estudio evidencia que la aproximación estadística que proponen diversos modelos basados en metodologías de corpus puede resultar de gran utilidad para obtener imágenes representativas de los patrones de variación de los marcadores discursivos a diferentes niveles. En este estudio nos hemos interesado particularmente por las dimensiones del 'registro', del 'género' y del 'estilo' -consideradas según la aproximación de Biber y Conrad (2011)-, y se ha podido comprobar que la metodología aplicada permite obtener resultados bastante clarificadores. Finalmente, y ligando con lo anterior, los resultados invitan a pensar que los postulados de la aproximación sociocognitiva y, particularmente el modelo que proponen Biber y Conrad (2011), podrían encontrar en los datos aportados por este trabajo un cierto soporte empírico.

No obstante, conviene tener presente que el estudio realizado ofrece solo una primera aproximación. Concretamente, tal y como se ha anotado en la discusión de los resultados, convendría indagar con mayor detalle cuáles son los criterios que condicionan la selección y uso concurrente de los marcadores discursivos más determinantes; y, también habría que analizar la validez jurilingüística de dichos usos. De hecho, la aparente coincidencia de algunos de los resultados obtenidos en este estudio con los del trabajo de Landone (2010), invitan a realizar trabajos contrastivos que podrían proporcionar herramientas de gran utilidad para las tareas de traducción y de redacción legislativa plurilingüe. De momento, este estudio ha permitido obtener algunos datos empíricos que permiten apuntar, más allá de la mera intuición, el carácter estratégico de los marcadores discursivos para garantizar la eficacia comunicativa y la eficiencia en la tarea de traducción de textos de este ámbito y, por lo tanto, para la calidad y la seguridad jurídica de los textos en euskera. Cabe presumir que, a partir de dichos datos, se podrá avanzar en estas investigaciones con un criterio más sólido y definido. Las catas exploratorias realizadas en esta dirección han resultado altamente alentadoras e invitan a seguir avanzando por este camino. 


\section{REFERENCIAS BIBLIOGRÁFICAS}

Aierbe, A. (2009). Birformulazio-estrategiak eta komunikagarritasuna administrazio testuetan. En P. Salaburu \& I. Ugarteburu (Eds.), Espezialitateko bizkerak eta terminologia jardunaldiak: espezialitate bizkeren didaktika eta komunikazioa [en línea]. Disponible en: http://tinyurl.com/aierbe

Alberdi, X. (2011a). Diskurtso-markatzaile berri bat: Hurrenez, hurren birformulatzailea. Anuario del Seminario de Filologia Vasca Julio de Urquijo (ASJU), XLV(45), 1, 301325.

Alberdi, X. (2011b). Erran nabi baita birformulatzaile esplikatiboa gaurko euskaran. Anuario del Seminario de Filología Vasca Julio de Urquijo (ASJU), XLV(45), 277304.

Alberdi, X. (Ed.) (2014). [en prensa] Birformulazioa eta birformulatzaileak euskaraz: Bilbao: Universidad del País Vasco/Euskal Herriko Unibertsitatea.

Alberdi, X. \& Ezeiza, J. (2013). Birformulazioa eta birformulatzaile esplikatiboak hizkera juridikoan. Euskera, 57(3), 607-657.

Aschenberg, H. \& Loureda, Ó. (2011). Introducción. Marcadores del discurso: Descripción, definición, contraste. En H. Aschenberg \& Ó. Loureda (Eds.), Marcadores del discurso: De la descripción a la definición (pp. 9-31). Frankfurt am Main: Iberoamericana-Vervuert.

Bhatia, A. \& Bhatia, V. (2011). Discursive Illusions in Legislative Discourse: A SocioPragmatic Study. International Journal for the Semiotics of Law, 24-1 [en línea]. Disponible en: http://www.springerlink.com/content/0952-8059/24/1/.

Bhatia, V. (1993). Analysing Genre-Language Use in Professional Settings. Londres: Longman.

Bhatia, V. (2002). Applied genre analysis: A multi-perspective model. IBÉRICA: Revista de la Asociacion Europea de Lenguas para Fines Especificos, 3-19.

Bhatia, V., Langton, N. M. \& Lung, J. (2004). Legal discourse: Opportunities and threats for corpus linguistics. En U. Connor \& T. A. Upton (Eds.), Discourse in the Professions. Perspectives from Corpus Linguistics (pp. 203-234). Ámsterdam: John Benjamins.

Biber, D. (1988). Variation across speech and writing. Cambridge: Cambridge University Press.

Biber, D. (1995). Dimensions of Register Variation: A Cross-Linguistic Perspective. Cambridge: Cambridge University Press. 
Biber, D. (2006). University Languages. A corpus-based study of spoken and written register. Ámsterdam: John Benjamins.

Biber, D. \& Finegan, E. (Eds.) (1994). Sociolinguistic perspectives on register. Oxford: Oxford University Press.

Biber, D. \& Conrad, S. (2011). Register, Genre and Style. Cambridge: Cambridge University Press.

Blakemore, D. (2002). Relevance and Linguistic Meaning: The Semantics and pragmatics of Discourse Markers. Cambridge: Cambridge University Press.

Cabré, M ${ }^{\text {a T. }}$ \& Gómez de Enterría, J. (2006). La enseñanza de los lenguajes de especialidad. La simulación global. Madrid: Gredos.

Casado, M. \& Barandiaran, A. (2011). Marcadores discursivos: Calas contrastivas en los reformuladores del español y el euskera. En. H. Aschenberg \& Ó. Loureda (Eds.), Marcadores del discurso: De la descripción a la definición (pp. 375396). Frankfurt am Main: Iberoamericana-Vervuert.

Ciapuscio, G. (2003). Textos especializados y terminología. Barcelona: IULA.

Conrad, S. \& Biber, D. (Eds.) (2001). Variation in English: Multi-dimensional studies. Londres: Longman.

Esnal, P. (2002). Testu-antolatzaileak. Hizpide, 50, 25-36.

Euskaltzaindia (Real Academia de la Lengua Vasca) (1990). Euskal Gramatika Lehen Urratsak-III (lokailuak). Bilbao: Euskaltzaindia.

Euskaltzaindia (Real Academia de la Lengua Vasca) (2000). Hið̧tegi Batua. Bilbao: Euskaltzaindia.

Euskaltzaindia (Real Academia de la Lengua Vasca) (2008). Testu-antolatraileak. Erabilera estrategikoa. Bilbao: Euskaltzaindia, Jagon Saila.

Euskaltzaindia (Real Academia de la Lengua Vasca) (2012). Euskaltraindiaren hiztegia. San Sebastián: Euskaltzaindia-Elkar-Elhuyar.

Ezeiza, J. (2011a). PLATAFORMA GARALEX: Infraestructura tecnológica para la investigación y la didáctica de lenguaje del ámbito de las ciencias jurídicas. En M. L. Carrió-Pastor, M. Candel \& A. Miguel (Eds.), Actas del III Congreso Internacional de Lingüistica del Corpus. Las tecnologías de la Información y las Comunicaciones: presente y futuro en el análisis del corpus (pp. 683-694). Valencia: UPV. 
Ezeiza, J. (2011b). Testu-antolatzaileak zientzia juridikoen alorreko testuetan erregistroen, generoen eta estilo-joeren bereizle. Hurbilpen estatistikoa. Informe de investigación inédito presentado para optar a la plaza AGC8L1-D00113-5 de profesor/a permanente de Lenguaje y Comunicación Jurídica en la Universidad del País Vasco.

Ezeiza, J. (2014). Zenbait lotura-hitz iragarleren erabilera Zuzenbideko testuetan (I): Baldin eta, salbu eta, baizik eta eta harik eta testu-antolatzaileen erabilera lege-testuetan eta testu akademikoetan: Aukera estilistikoak eta itzulpenestrategiak. ELERIA, 24, 77-97.

Garcés, $M^{a}$ P. (2008). La organización del discurso: Marcadores de ordenación y de reformulación. Madrid: Iberoamericana-Vervuert.

Gémar, J. C. (Dir.) (1982). Langage du droit et traduction: Essais de jurilinguistique/The language of the law and translation: Essays on jurilinguistics. Québec: Linguatech.

Gémar, J. C. \& Kasirer, N. (Eds.) (2005). Jurilinguistique: Entre langues et droits / Jurilinguistics: Between law and language. Montréal: Éditions Thémis.

Landone, E. (2010). Notas en torno a los marcadores del discurso en la normativa territorial de las Comunidades Autónomas españolas. En: L. Chierichetti \& G. Garofalo (Eds.), Lengua y Derecho: Lineas de investigación interdisciplinaria (pp. 35-58). Bern: Peter Lang.

Larringan, L. M. (1996). Testu-antolatzaileak bi testu motatan: Testu informatiboa eta argudiapen-testua. Tesis doctoral, Universidad del País Vasco (UPV/EHU) Vitoria-Gasteiz.

Loureda, Ó. \& Acín, E. (2010). Cuestiones candentes en torno a los marcadores del discurso en español. En Ó. Loureda \& E. Acín (Coords.), Los estudios sobre marcadores del discurso en español, boy (pp. 7-60). Madrid: Arco/Libros.

Lozano, J. (Coord.) (1997). Argiro idazteke proposamenak eta ariketak. Vitoria-Gasteiz: IVAP-Servicio Editorial del Gobierno Vasco.

Martí Sánchez, M. (2011). Los conectores discursivos (entre los otros marcadores discursivos y los otros conectores). En A.M. Cestero \& M. Martí Sanchez (Coords.), Lingüistica en la red, monográfico: I Jornadas de lengua y comunicación: marcadores discursivos. [en línea]. Disponible en http://www.linred.es/numero9_ articulo_8.html

Martín Zorraquino, Ma A. \& Portolés, J. (1999). Los marcadores del discurso. En I. Bosque \& V. Demonte (Eds.), Gramática descriptiva de la lengua española (pp. 4051-4213). Madrid: Espasa. 
Mazzi, D. (2011). 'In Other Words,...': A corpus-based Study of Reformulations in Judicial Discourse. Hermes, Journal of Language and Communication Studies, 46, 11-24.

Parodi, G. (2010). Lingüistica del corpus: De la teoría a la empiria. Madrid: Iberoamericana.

Portolés, J. (2007). Marcadores del discurso. Barcelona: Ariel.

Real Academia Española (RAE)/Asociación de Academias de la Lengua Española (2010). Nueva gramática de la lengua española, Sintaxis II. Madrid: Espasa Libros.

Reppen, R., Fitzmaurice, S. M. \& Biber, D. (2002). Using corpora to explore linguistic variation. Ámsterdam: John Benjamins.

Salaburu, P. \& Alberdi, X. (Eds.) (2012). The Challenge of a Bilingual Society in the Basque Country. Reno (Nevada): Center for Basque Studies, University of Nevada (Reno); Universidad del País Vasco/Euskal Herriko Unibertsitatea.

Urrutia, A. (2005). Euskararen jurilinguistika: Alor berria ote? AVD-ZE A, 7, 93-104.

Urrutia, A. (2008). Legeen eta Administrazioaren hizkera testu-antolatzaileen ikuspegitik. Euskera, LIII(2), 525-546.

Vázquez Veiga, N. (2011). El tratamiento lexicográfico de los marcadores del discurso. En A.M. Cestero \& M. Martí Sánchez Coords.), I Jornadas de lengua y comunicación: Marcadores discursivos. [en línea]. Disponible en http://www.linred. es/monograficos_pdf/LR_monografico9_articulo5.pdf 


\section{NOTAS}

1 El año 2013 los juzgados de la Comunidad Autónoma del País Vasco redactaron en euskera un total de 3.822.508 documentos. Ello constituye el 62,56\% de los documentos emitidos.

2 Mostramos a continuación algunas de ellas: 'enlaces extraoracionales/supraoracionales/ textuales', 'conectores', 'conectores pragmáticos/discursivos/textuales/argumentativos', 'conectivos', 'operadores discursivos', 'particulas discursivas', 'ordenadores del discurso'...

3 "El significado procedimental, también llamado de procesamiento, es la información sobre cómo procesar el significado conceptual, el cual se define a su vez como la información léxica sobre el contenido proposicional de los enunciados" (Loureda \& Acín, 2010: 35).

4 Se trata de un reconocimiento que exige a los poderes públicos que los textos y comunicaciones que generen puedan ser comprendidos sin dificultades por la población interesada. Se trataría de un derecho reconocido y desarrollado, entre otros documentos, en el Código Europeo de Buena Conducta Administrativa, en la Directiva 2010/64/UE relativa al derecho a interpretación y a traducción en los procesos penales, en la Directiva 2012/13/UE relativa al derecho a la información en los procesos penales, en la Directiva 2012/29/UE por la que se establecen normas mínimas sobre los derechos, el apoyo y la protección de las víctimas de delitos, en La Carta de Derechos del Ciudadano ante la Justicia, en el Plan Estratégico para la Modernización del sistema de Justicia, en el Posicionamiento Integral para la Modernización y Euskaldunización de la Administración de Justicia en el País Vasco o en el Plan para la Modernización del Lenguaje Jurídico.

5 Resultado de los proyectos DB (OTRI 2007.0077), EHLB (OTRI: 2008.0368) e HIZLAN (DIPE08/16). En http://www.hizlan.org/db-zuzenbidea

6 Para el desarrollo del la plataforma Web que da soporte al gestor de corpus HIZLAN se contó con la inestimable contribución de Josu Landa y Eusebi Calonge de la Unidad I+D empresarial, Ametzagaiña A.I.E. [URL: http://www.ametza.com/castellano/ametza.htm], centro de investigación y desarrollo en nuevas tecnologías, con el que se suscribió un proyecto de colaboración para realizar dicha plataforma.

7 http://www.ehu.es/ehg/zuzenbidea/

8 http://www.ehu.es/euskara-orria/euskara/ereduzkoa/

9 http://ehu.es/ehg/goenkale/

10 http://ehu.es/ehg/pkc/

11 http://www.ehu.es/ehg/zio/

12 http://www.ztcorpusa.net/aurkezpena.htm

13 Dicho informe se presentó en el concurso-oposición para la provisión de la plaza de profesor/a permanente de Lenguaje y Comunicación Jurídica en la Facultad de Derecho de la Universidad del País Vasco (Código: AGC8L1-D00113-5). 